Ann. Zootech., I974, 23 (2), I7I-I84.

\title{
INCORPORATION DE PROPORTIONS VARIABLES DE MATIÈRES GRASSES (HUILE D'ARACHIDE) DANS LE RÉGIME DU PORC EN CROISSANCE-FINITION, EN RELATION AVEC LE TAUX DE MATIÈRES AZOTÉES
}

\author{
II. - INFLUENCE SUR LES PERFORMANCES DE CROISSANCE \\ ET ILA COMPOSITION CORPORELILE
}

\author{
Y. HENRY \\ avec la collaboration technique de H. Bousquet, R. Kerisit et L. Barrière \\ Station de Recherches sur l'Élevage des Porcs, \\ Centre national de Recherches zootechniques, I. N.R.A., \\ 78350 Jouy en Josas
}

\section{RÉSUMÉ}

$\mathrm{Au}$ cours d'une expérience portant sur 60 porcs en croissance de race Large White, entre 25 et $90 \mathrm{~kg}$ de poids vif, nourris à volonté de régimes semi-purifiés à base d'amidon de maïs et renfermant i2 ou i6 p. Ioo de protéines de poisson, sous forme de farine de hareng de Norvège, il a été procédé à l'étude de l'influence de taux croissants d'huile d'arachide (2, I 2 et 22 p. IOo) dans la ration sur la consommation de nourriture, les performances de croissance, l'efficacité alimentaire et les caractéristiques de composition corporelle à l'abattage. Six lots de ro animaux (moitié mâles castrés, moitié femelles) étaient mis en comparaison suivant un dispositif factoriel $3 \times 2$.

Les résultats montrent que l'élévation de la concentration en énergie du régime, d'un peu plus de 34 oo kcal d'énergie digestible par kilo à près de 4200 après addition d'huile d'arachide, provoque, quel que soit le taux azoté, une diminution systématique de la consommation quotidienne d'aliment ; mais le taux de diminution observé est plus faible que celui de l'augmentation de la valeur énergétique du régime, de sorte que le niveau d'ingestion d'énergie est légèrement accru ( +12 p. Ioo à I 2 p. Ioo de matières azotées). Au niveau de la croissance, il en résulte une amélioration du gain moyen pondéral, accompagnée d'une augmentation de l'état d'engraissement des carcasses, favorisé par ailleurs par un apport accru d'acides gras longs. Il est à noter toutefois une interaction entre le taux de farine de hareng et celui d'huile d'arachide : l'accroissement de ce dernier en présence d'un pourcentage plus élevé de protéines de poisson exerce un effet dépressif sur la consommation, et par suite sur la vitesse de croissance. D'après l'évolution de la consommation d'énergie en fonction du poids vif, il apparaît que les femelles ajustent mieux que les mâles castrés leur niveau d'ingestion quand varie la concentration en énergie du régime. La consommation moyenne, en Mcal d'énergie digestible, par kilo de poids métabolique ( $\left.\mathrm{P}^{075}\right)$, est plus élevée chez les mâles castrés que chez les femelles $\left(0,4\right.$ I contre $\left.0,3^{8}\right)$. 


\section{INTRODUCTION}

Étudiant les effets combinés des variations des taux d'incorporation de matières grasses, sous forme d'huile d'arachide, et de matières azotées dans des régimes semipurifiés à base d'amidon de maïs et de farine de hareng de Norvège, chez le Porc en croissance-finition, nous avons rapporté dans un travail précédent (HENRY et DE WILDE, I973) les résultats concernant l'utilisation digestive des substances énergétiques et azotées de la ration, ainsi que la rétention azotée, dans le cas d'une alimentation égalisée en matière sèche. En dehors de l'effet dépressif exercé par l'élévation du pourcentage d'huile d'arachide de 2 à $22 \mathrm{p}$. Ioo sur le coefficient d'utilisation digestive de l'énergie, principalement pendant la phase initiale de l'expérimentation, il a été observé qu'un apport accru d'énergie entraîne une amélioration de la rétention azotée à partir d'un certain niveau de 1'apport azoté. Ces résultats obtenus en cages à métabolisme ont été complétés, suivant le même schéma expérimental, par un essai en lots, dans le but d'étudier l'influence de la concentration en énergie du régime, après incorporation d'huile d'arachide, sur la consommation de nourriture, les performances de croissance, l'efficacité alimentaire et les caractéristiques de composition corporelle. Des résultats partiels de cette étude ont fait l'objet d'une publication antérieure (HENRY, I966).

\section{MATÉRIEL，ET MÉTHODES}

Soixante porcelets, de race Large White et d'un poids moyen initial de $25 \mathrm{~kg}$, sont répartis entre 6 lots de Io animaux ( 5 mâles castrés et 5 femelles), soumis à une variation de la concentration en énergie et đu taux de matières azotées dans la ration, suivant un plan factoriel $3 \times 2$ :

- 3 taux de matières grasses : 2, I 2 et 22 p. Ioo d'huile d'arachide, qui a été choisie surtout en fonction des commodités de mélange avec les autres composants de la ration;

- 2 taux de protéines de poisson : i 2 et i6 p. Ioo.

TABLEAU I

Résultats moyens d'analyse et valeur énergétique des régimes

\begin{tabular}{|c|c|c|c|c|c|c|}
\hline M. azotées (p. 100) & \multicolumn{3}{|c|}{12} & \multicolumn{3}{|c|}{16} \\
\hline H. d'arachide (p. 100) & 2 & 12 & 22 & 2 & 12 & 22 \\
\hline Lot & 1 & 2 & 3 & 4 & 5 & 6 \\
\hline Mat. sèche (p. 100) & 91,7 & 92,3 & 92,9 & 91,5 & 91,6 & 93,0 \\
\hline Mat. azotées (p. 100) & 11,9 & 12,4 & 12,0 & 16,0 & 15,8 & 16,0 \\
\hline Énergie digestible (kcal/kg) & $3411^{\circ}$ & 3745 & 4137 & 3533 & 3805 & 4233 \\
\hline Mat. azot. digestibles (p. 100). & 10,3 & 10,7 & $10,,^{\prime}$ & 14,2 & 14,1 & 14,0 \\
\hline
\end{tabular}


Des couples d'animaux de même sexe et provenant d'une même portée, sont affectés aux 6 traitements, selon un dispositif en blocs incomplets équilibrés (CoCHRAN et CoX, I962), à raison d'une série de 5 répétitions par sexe.

Les régimes, de type semi-purifié, renferment, comme seule source azotée, une farine de hareng de Norvège, aux taux respectifs de 16 p. Ioo dans les lots $\mathrm{I}, 2$ et 3 (à 12 p. roo de matières azotées) et 2 I, 5 p. Ioo dans les lots 4, 5 et 6 (à I 6 p. I oo de matières azotées). En plus de l'huile d'arachide (respectivement 2 , I 2 et 22 p. Ioo dans les lots I-3, 2-4 et 3-6), on y introcluit 20 p. Ioo de sucre dénaturé, ro p. Ioo de cellulose de bois purifiée, de nature fibreuse, 5 p. Ioo d'un mélange minéral et des vitamines, le complément de la ration étant fourni par l'amidon de maïs. Les détails concernant les caractéristiques des matières premières et des régimes ont été rapportés précédemment (HenRy et De WiLde, I973). Dans le tableau i figurent les résultats moyens d'analyse des régimes, ainsi que leur valeur énergétique, estimée à partir de l'essai de digestibilité.

Les aliments sont đistribués sous forme humide, à raison de 2 parties d'eau pour une partie d'aliment sec, au cours de 3 repas journaliers et selon l'appétit; le complément d'eau est fourni à discrétion entre les repas. Les consommations sont enregistrées quotidiennement.

Les animaux sont pesés tous les $\mathrm{I} 4$ jours. A $90 \mathrm{~kg}$, ils sont abattus et, après un ressuyage de 24 heures, leurs carcasses sont soumises à la découpe parisienne, en complément des mensurations habituelles (épaisseur du lard au dos et au rein, longueur de la carcasse).

Les résultats de l'expérience sont traités par la technique habituelle d'analyse de variance d'un plan factoriel, avec séparation des effets principaux et d'interaction.

\section{RÉSULTATS}

Compte tenu du type de dispositif expérimental choisi (blocs incomplets équilibrés), les résultats de l'expérience ont été soumis dans un premier temps à une analyse de variance particulière, permettant de corriger les effets des traitements à partir de la variation entre blocs ou couples d'animaux (Cochran et Cox, I962). L'exploitation des données selon ce dispositif n'a fait ressortir aucun avantage sur celui des blocs complets, c'est-à-dire après élimination de l'effet " couples ". C'est ainsi que 1'efficacité du premier dispositif par rapport au second, estimée d'après FISChER (1958), est de I38 et I24 p. roo respectivement pour le gain de poids vif moyen journalier et l'indice de consommation, entre 25 et $60 \mathrm{~kg}$ de poids vif. Pour ce qui est des caractéristiques de composition corporelle, elle n'est plus que de II7 et I Io p. Ioo respectivement pour les pourcentages de jambon + longe et de bardière + panne dans la carcasse ; elle est nulle en ce qui concerne l'épaisseur moyenne du lard dorsal. Dans ces conditions, nous avons procédé, pour chacun des critères, à une analyse de variance suivant le schéma en blocs " randomisés " complets.

\section{A. - Croissance, consommation et efficacité alimentaire}

En raison de l'absence d'interaction entre le sexe et les traitements au niveau des différents critères, seuls sont rapportés les résultats moyens (mâles castrés, femelles) pour chacun des 6 lots, d'abord pendant la première partie de la croissance, jusqu'à $60 \mathrm{~kg}$ de poids vif (tabl. 2), puis pendant toute la durée de l'expérience (tabl. 3).

a) Résultats entre 25 et $60 \mathrm{~kg}$.

Les variations du gain moyen journalier font apparaitre une interaction significative entre la concentration en matières grasses et le pourcentage de matières azotées. L'élévation de la proportion d'huile d'arachide de 2 à 22 p. Ioo entraîne une 
légère amélioration de la vitesse de croissance, au taux de $\mathrm{I} 2 \mathrm{p}$. Ioo de matières azotées ; au taux de 16 p. Ioo, au contraire, il s'ensuit une nette dépression du gain moyen journalier. Cette différence de réponse en fonction du taux d'huile d'arachide

\section{TABLEAU 2}

Résultats moyens de croissance et de consommation entre 25 et $61 \mathrm{~kg}$ de poids vif

\begin{tabular}{|c|c|c|c|c|c|c|}
\hline \multirow[b]{2}{*}{$\begin{array}{l}\text { Mat. azotées } \\
\text { (p. 100) }\end{array}$} & \multirow{2}{*}{$\begin{array}{c}\text { Huile } \\
\text { d'arachide } \\
\text { (p. 100) }\end{array}$} & \multirow{2}{*}{$\begin{array}{c}\text { Gain } \\
\text { de poids vif } \\
(\mathrm{g} / \mathbf{j})\end{array}$} & \multicolumn{2}{|c|}{ Consommation par jour } & \multicolumn{2}{|c|}{ Indice de consommation } \\
\hline & & & $\mathrm{kg}$ aliment & ED, Mcal & $\begin{array}{c}\mathrm{kg} \\
\underset{\text { gain }}{\operatorname{aliment} / \mathrm{kg}}\end{array}$ & $\begin{array}{c}\mathrm{ED}, \\
\mathrm{Mcal} / \mathrm{kg} \text { gain }\end{array}$ \\
\hline 12 & $\begin{array}{r}2 \\
12 \\
22\end{array}$ & $\begin{array}{l}574 \\
591 \\
602\end{array}$ & $\begin{array}{l}1,78 \\
1,67 \\
1,66\end{array}$ & $\begin{array}{l}6,09 \\
6,25 \\
6,88\end{array}$ & $\begin{array}{l}3,11 \\
2,81 \\
2,77\end{array}$ & $\begin{array}{l}10,60 \\
10,63 \\
11,45\end{array}$ \\
\hline 16 & $\begin{array}{r}2 \\
12 \\
22\end{array}$ & $\begin{array}{l}674 \\
621 \\
597\end{array}$ & $\begin{array}{l}1,84 \\
1,70 \\
1,53\end{array}$ & $\begin{array}{l}6,51 \\
6,45 \\
6,50\end{array}$ & $\begin{array}{l}2,76 \\
2,76 \\
2,62\end{array}$ & $\begin{array}{r}9,74 \\
10,49 \\
11,11\end{array}$ \\
\hline \multicolumn{2}{|c|}{ Analyse statistique $\left(^{1}\right)$} & $22,4(11,6)$ & $0,053(9,8)$ & $0,20(9,9)$ & $0,058(6,6)$ & $0,22(6,6)$ \\
\hline $\begin{array}{r}\text { Effets p } \\
\text { valeurs } \\
\text { Sexe }\left\{\begin{array}{l}\text { mâles } \\
\text { femell }\end{array}\right.\end{array}$ & $\begin{array}{l}\text { incipaux } \\
\text { moyennes } \\
\ldots \ldots \ldots \ldots \\
\text { s............. }\end{array}$ & $\begin{array}{l}631 * \\
588^{*}\end{array}$ & $\begin{array}{l}1,77^{* *} \\
1,62^{* *}\end{array}$ & $\begin{array}{l}6,74 * * \\
6,16 * *\end{array}$ & $\begin{array}{l}2,82 \\
2,79\end{array}$ & $\begin{array}{l}10,73 \\
10,61\end{array}$ \\
\hline M. azotées ( & o) $\left\{\begin{array}{l}12 \ldots \ldots \\
16 \ldots\end{array}\right.$ & $\begin{array}{l}589^{*} \\
631^{*}\end{array}$ & $\begin{array}{l}1,71 \\
1,69\end{array}$ & $\begin{array}{l}6,41 \\
6,49\end{array}$ & $\begin{array}{l}2,90 * * \\
2,71 * *\end{array}$ & $\begin{array}{l}10,89^{*} \\
10,44^{*}\end{array}$ \\
\hline H. d'arachid & $(\%)\left\{\begin{array}{r}2 \ldots \\
12 \ldots \\
22 \ldots\end{array}\right.$ & $\begin{array}{l}624 \\
606 \\
600\end{array}$ & $\begin{array}{l}1,81 \\
1,68 \mathrm{E}_{\mathrm{L}} * * \\
1,60\end{array}$ & $\begin{array}{l}6,30 \\
6,35 \mathrm{~F}_{\mathrm{L}}(0,10) \\
6,69\end{array}$ & $\begin{array}{l}2,93 \\
2,80 \mathrm{E}_{\mathrm{L}} * * \\
2,70\end{array}$ & $\begin{array}{l}10,17 \\
10,55 \mathrm{E}_{\mathrm{L}} * * \\
11,28\end{array}$ \\
\hline $\begin{array}{r}\text { Effets } d^{\prime} i \\
\mathrm{~N} \times \\
\mathrm{N} \times \\
\mathrm{N} \times\end{array}$ & $\begin{array}{l}\text { teraction } \\
\mathrm{E} \\
\mathrm{E}_{\mathrm{L}} \\
\mathrm{E}_{\mathrm{Q}}\end{array}$ & * & $(0,10)$ & $(0,10)$ & $(0,10)$ & \\
\hline
\end{tabular}

(1) $s_{\bar{x}}$ : écart-type de la moyenne (10 animaux par lot); entre parenthèses, coefficient de variation. Effets : $\mathrm{E}$ : taux d'huile d'arachide; $\mathrm{E}_{\mathrm{L}}$ : terme linéaire; $\mathrm{E}_{\mathrm{Q}}$ : terme quadratique. $\mathrm{N}$ : taux azoté. $\mathbf{N} \times \mathbf{E}$ : interaction taux azoté $\times$ taux d'huile d'arachide.

Seuils significatifs : ${ }^{* *}: 0,01 ; *: 0,05 ;(0,10): 0,10$.

Ces indications sont valables pour les tableaux suivants.

se retrouve d'ailleurs au niveau de la consommation journalière; cette dernière diminue beaucoup plus fortement avec les régimes à $\mathrm{I} 6 \mathrm{p}$. Ioo de matières azotées (- I7 p. IOo) qu'avec ceux à I2 p. Ioo (-7 p. IOo). Compte tenu des écarts observés dans la valeur énergétique des régimes $(+20 \mathrm{p}$. Ioo entre 2 et $22 \mathrm{p}$. Ioo d'huile d'arachide), on voit que la consommation journalière, exprimée en énergie digestible, 
n'est pratiquement pas modifiée par le taux d'huile d'arachide dans les lots à $\mathbf{I} 6$ p. Ioo de matières azotées; par contre, dans les lots à I 2 p. Ioo de matières azotées, elle augmente avec le taux d'incorporation de matières grasses, soit $\mathbf{2} 2 \mathrm{p}$. Ioo entre 2 et 22 p. roo d'huile d'arachide.

TABLEAU 3

Résultats moyens de croissance et de consommation entre 25 et $92 \mathrm{~kg}$ de poids vif

\begin{tabular}{|c|c|c|c|c|c|c|}
\hline \multirow{2}{*}{$\begin{array}{c}\text { Mat. azotées } \\
\text { (p. 100) }\end{array}$} & \multirow{2}{*}{$\begin{array}{c}\text { Huile } \\
\text { d'arachide } \\
\text { (p. 100) }\end{array}$} & \multirow{2}{*}{$\begin{array}{c}\text { Gain } \\
\text { de poids vif } \\
(\mathrm{g} / \mathrm{j})\end{array}$} & \multicolumn{2}{|c|}{ Consommation par jour } & \multicolumn{2}{|c|}{ Indice de consommation } \\
\hline & & & $\mathrm{kg}$ aliment & ED, Mcal & $\begin{array}{l}\mathbf{k g} \\
\underset{\text { aliment } / \mathrm{kg}}{\text { gain }}\end{array}$ & $\begin{array}{c}\text { ED, } \\
\text { Mcal/kg gain }\end{array}$ \\
\hline 12 & $\begin{array}{r}2 \\
12 \\
22\end{array}$ & $\begin{array}{l}671 \\
661 \\
672\end{array}$ & $\begin{array}{l}2,19 \\
1,97 \\
1,93\end{array}$ & $\begin{array}{l}7,48 \\
7,37 \\
7,97\end{array}$ & $\begin{array}{l}3,26 \\
2,98 \\
2,87\end{array}$ & $\begin{array}{l}11,14 \\
11,17 \\
11,89\end{array}$ \\
\hline 16 & $\begin{array}{r}2 \\
12 \\
22\end{array}$ & $\begin{array}{l}732 \\
689 \\
649\end{array}$ & $\begin{array}{l}2,26 \\
2,04 \\
1,84\end{array}$ & $\begin{array}{l}7,99 \\
7,77 \\
7,80\end{array}$ & $\begin{array}{l}3,10 \\
2,97 \\
2,87\end{array}$ & $\begin{array}{l}10,95 \\
11,31 \\
12,13\end{array}$ \\
\hline Analyse & tatistique & $23,9(11,1)$ & $0,056(8,7)$ & $0,21(8,7)$ & $0,062(6,5)$ & $0,24(6,7)$ \\
\hline $\begin{array}{l}\text { Effets pr } \\
\text { Valeurs }\end{array}$ & $\begin{array}{l}\text { incipaux } \\
\text { noyennes }\end{array}$ & & & & & \\
\hline Sexe $\left\{\begin{array}{l}\text { mâles } \\
\text { femell }\end{array}\right.$ & $\begin{array}{l}\text { castrés .... } \\
\text { s } \ldots . . . .\end{array}$ & $\begin{array}{l}700^{*} \\
658^{*}\end{array}$ & $\begin{array}{l}2,12^{* *} \\
1,96 * *\end{array}$ & $\begin{array}{l}8,03^{* *} \\
7,43^{* *}\end{array}$ & $\begin{array}{l}3,03 \\
2,98\end{array}$ & $\begin{array}{l}11,53 \\
11,33\end{array}$ \\
\hline M. azotées \{ & $\begin{array}{l}12 \ldots \ldots \\
16 \ldots \ldots \\
16 \ldots\end{array}$ & $\begin{array}{l}668 \\
690\end{array}$ & $\begin{array}{l}2,03 \\
2,05\end{array}$ & $\begin{array}{l}7,61 \\
7,85\end{array}$ & $\begin{array}{l}3,04 \\
2,98\end{array}$ & $\begin{array}{l}11,40 \\
11,46\end{array}$ \\
\hline H. d'arachide & $(\%)\left\{\begin{array}{r}2 . \\
12 . \\
22 .\end{array}\right.$ & $\begin{array}{lll}701 & & \\
675 & \mathrm{E}_{\mathrm{L}} & (0,10) \\
661 & & \end{array}$ & $\begin{array}{l}2,23 \\
2,01 \mathrm{E}_{\mathrm{L}} * * \\
1,88\end{array}$ & $\begin{array}{l}7,73 \\
7,57 \\
7,88\end{array}$ & $\begin{array}{l}3,18 \\
2,98 \mathrm{E}_{\mathrm{L}}{ }^{* *} \\
2,87\end{array}$ & $\begin{array}{l}11,04 \\
11,24 E_{L} * * \\
12,01\end{array}$ \\
\hline $\begin{array}{r}\text { Effets } d \\
\qquad \begin{array}{l}\mathrm{N} \times \\
\mathrm{N} \times \\
\mathrm{N} \times\end{array}\end{array}$ & $\begin{array}{l}\text { nteraction } \\
\mathrm{E} \\
\mathrm{E}_{\mathrm{L}} \\
\mathrm{E}_{\mathrm{Q}}\end{array}$ & $(0,10)$ & & & & \\
\hline
\end{tabular}

Les variations de l'indice de consommation, en $\mathrm{kg}$ d'aliment par $\mathrm{kg}$ de gain, en fonction de la concentration en énergie du régime sont évidentes. Exprimé en méga. calories d'énergie digestible par $\mathrm{kg}$ de gain, l'indice de consommation est d'autant plus élevé que le taux d'incorporation d'huile d'arachide est lui-même plus important (de 6,3 à 6,7 entre 2 et 22 p. Ioo d'huile d'arachide, soit +6 p. I00).

L'augmentation du taux de matières azotées de $\mathrm{I} 2$ à $\mathrm{I} 6 \mathrm{p}$. roo exerce une influence favorable sur le gain moyen journalier, qui passe de 589 à $63 \mathrm{I} \mathrm{g}$, et l'indice de consommation, qui décroît de I0,9 à ro,4 Mcalories d'énergie digestible par $\mathrm{kg}$ de gain.

L'influence du sexe fait ressortir les observations habituelles, à savoir une vitesse de croissance et une consommation d'énergie plus faibles chez les femelles que chez les mâles castrés, tandis que l'indice de consommation demeure inchangé. 
b) Résultats entre 25 et $92 \mathrm{~kg}$.

Sur la période totale de croissance, les résultats obtenus sont comparables à ceux observés en début de croissance, tout au moins en ce qui concerne les effets du taux d'huile d'arachide et l'interaction entre ce dernier et le taux de matières azotées. Nous retrouvons ici encore, sous l'influence du pourcentage de matières grasses, une dépression plus forte du niveau d'ingestion avec les régimes à $I 6 \mathrm{p}$. Ioo de matières azotées (- I9 p. I00) qu'avec ceux à I2 p. IOO (- I2 p. I00). Exprimée en énergie digestible, la consommation augmente avec la concentration en énergie à I $2 \mathrm{p}$. Ioo de matières azotées, tandis qu'à 16 p. Ioo elle n'est pas modifiée. Par ailleurs, l'augmentation de la proportion d'huile d'arachide entraîne une détérioration de l'indice de consommation, exprimé en Mcalories d'énergie digestible par kg de gain (de II,o à I 2,0 respectivement pour 2 et 22 p. Ioo de matières grasses).

En ce qui concerne le niveau azoté, au contraire, on ne constate cette fois aucune influence significative sur la vitesse de croissance ni sur l'indice de consommation : ceci résulte de l'absence de réponse à une élévation du taux de matières azotées de I 2 à $\mathbf{6} \mathrm{p}$. Ioo pendant la phase terminale de la croissance (au-delà de $60 \mathrm{~kg}$ de poids vif).

c) Évolution de la croissance et de la consommation: velation entre la consommation d'énergie et le poids vif.

L'évolution du gain de poids vif en fonction de l'âge (fig. I) fait de nouveau ressortir une interaction entre le taux d'huile d'arachide et celui de matières azotées : au taux de matières azotées le plus élevé ( 16 p. IOo), la croissance maximum est

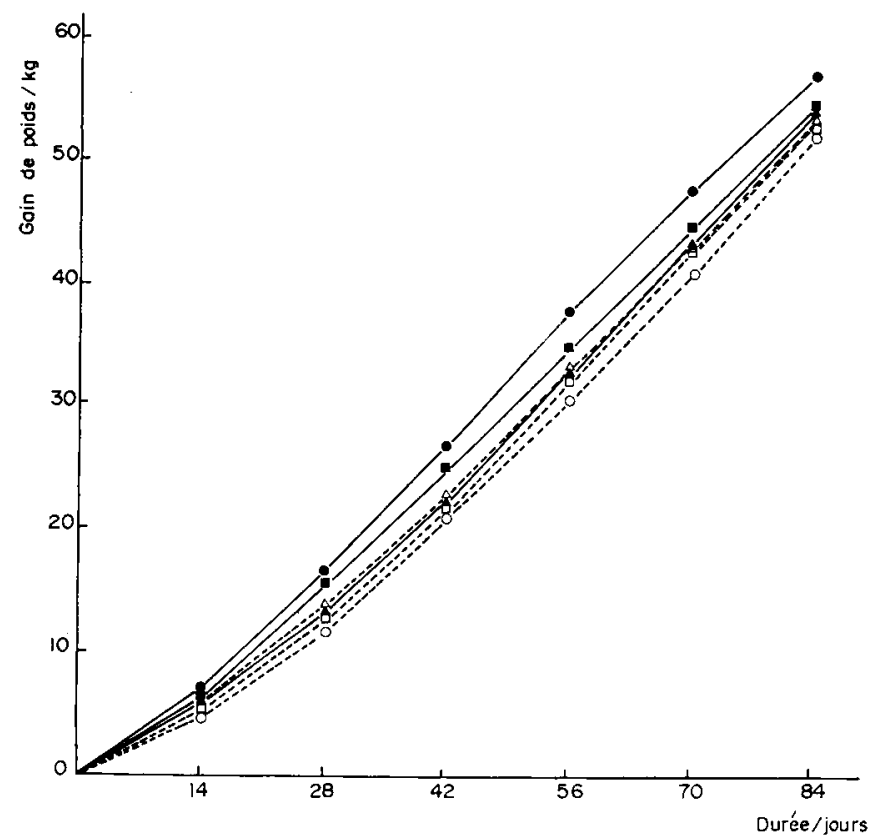

FIG. I. - Evolution du gain de poids

H. arachide (p. IOO) 2 I2 22

Nat. azot. $\left\{\begin{array}{llll}12 & \ldots \ldots & 0 & \square\end{array}\right.$

(p. I00) $\left\{\begin{array}{lll}16 & \bullet\end{array}\right.$ 
obtentue à l'aide du régime le moins pourvu en huile d'arachide ( $2 \mathrm{p}$. IOO), alors qu'au taux le plus faible ( 2 p. Ioo) la croissance pondérale est peu modifiée par la concentration en matières grasses.

Dans les figures 2 et 3 , sont rapportées les variations de la consommation journalière d'énergie, exprimée en Mégacalories d'énergie digestible par lot et par quinzaine, en fonction du poids vif, respectivement pour les mâles castrés et les femelles.

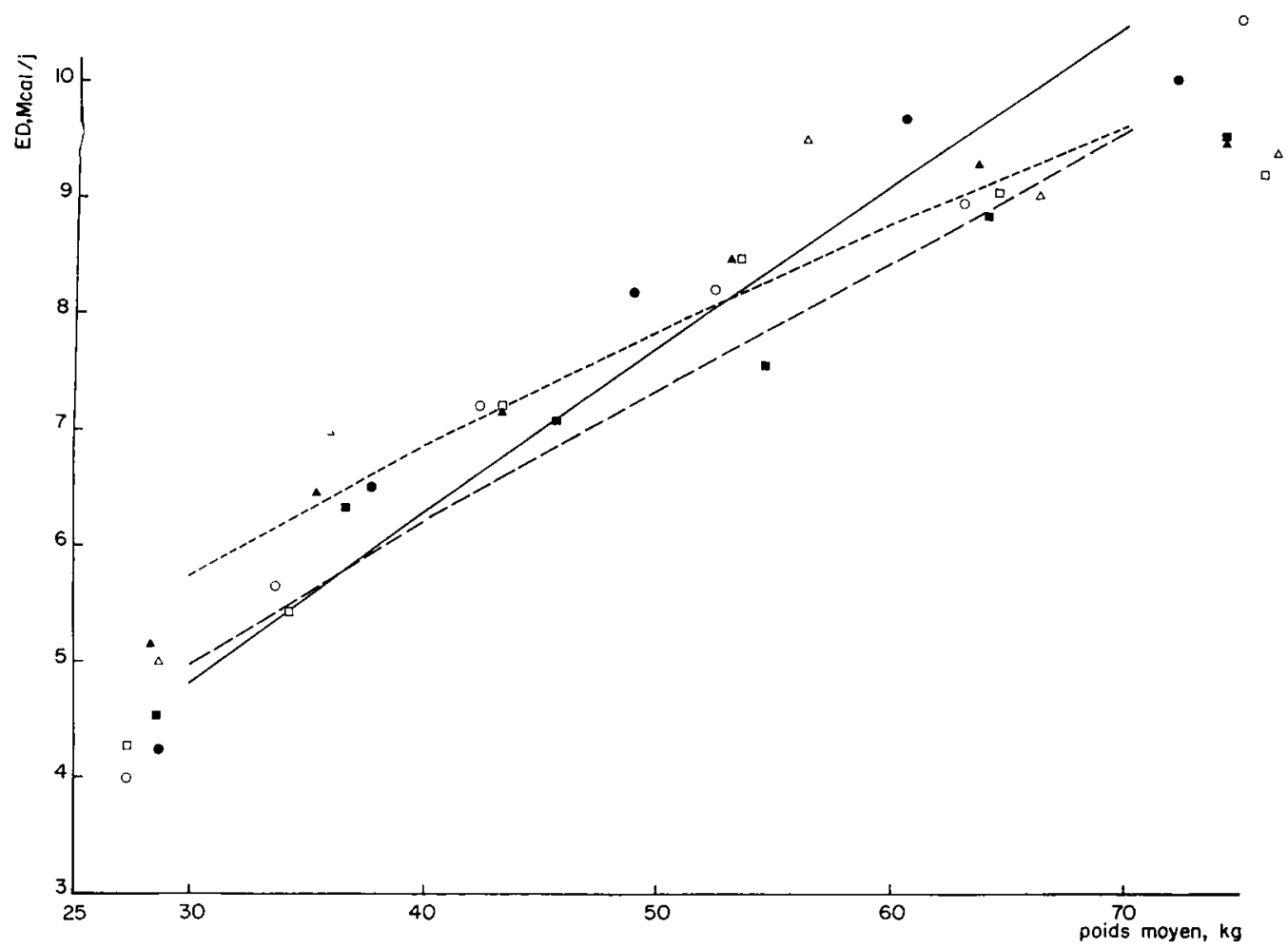

FIG. 2.- Évolution de la consommation d'énergie en fonction du poids vif moyen par quinzaine chez les mâles castrés

M. azotées (p. Ioo) I 2 I6

$\underset{\text { (p. Ioo) }}{\operatorname{arachide}}\left\{\begin{array}{cccc}2 & & 0 & \bullet \\ 12 & -\ldots \ldots \ldots & \square & \Delta \\ 22 & \ldots \ldots \ldots & \bullet\end{array}\right.$

Dans les deux cas, on observe une évolution curvilinéaire, ce qui nous a conduit à comparer les droites de régression entre lots, après transformation logarithmique des données. En l'absence d'effet du taux de matières azotées, les données ont été regroupées en fonction du taux de lipides. Les résultats du tableau 4 montrent que, chez les mâles castrés, le coefficient de régression diminue à mesure qu'augmente le pourcentage d'huile d'arachide, alors que chez les femelles, il ne diffère pas significativement d'un lot à 1'autre. I1 en est de même de 1'ordonnée à l'origine, qui augmente avec le taux d'huile d'arachide. Qu'il s'agisse des mâles castrés ou des femelles, la comparaison des moyennes ajustées après régression ne fait apparaître aucune différence significative entre les taux de matières grasses. De ces observations, il 


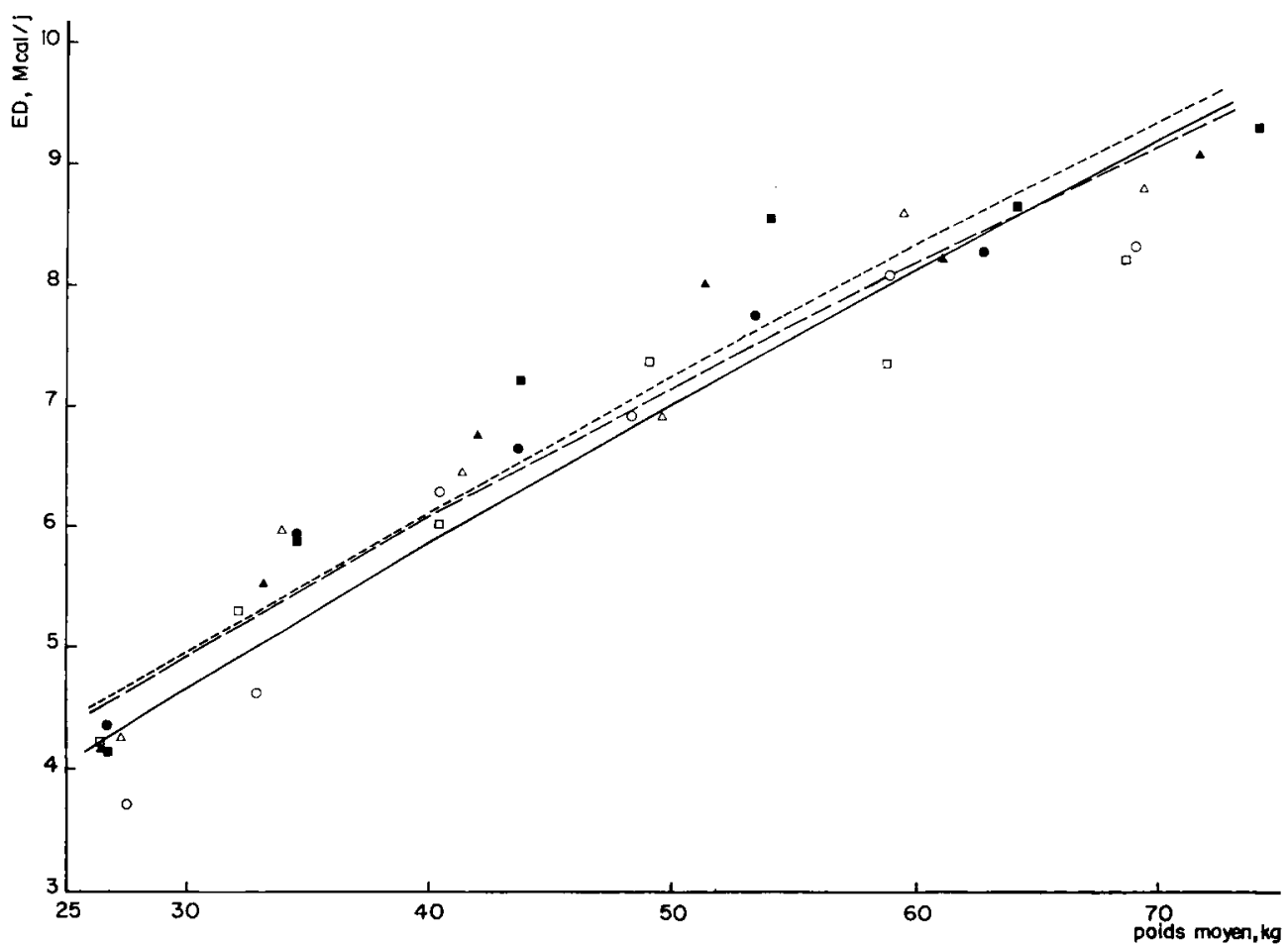

FIG. 3. - Évolution de la consommation d'ênergie en fonction du poids vif moyen par quinzaine chez les femelles

M. azotées (p. Iо0) I2 16

H. $\underset{\text { (p. I0o) }}{\operatorname{arachide}}\left\{\begin{array}{rlll}2 & - & 0 & \bullet \\ \mathrm{I} 2 & ---1 & \square & \Delta\end{array}\right.$

TABLEAU 4. - Comparaison des paramètres des équations de régression reliant la consommation d'énergie (ED, Mcal/j) et le poids vif (kg), après transformation logarithmique des données

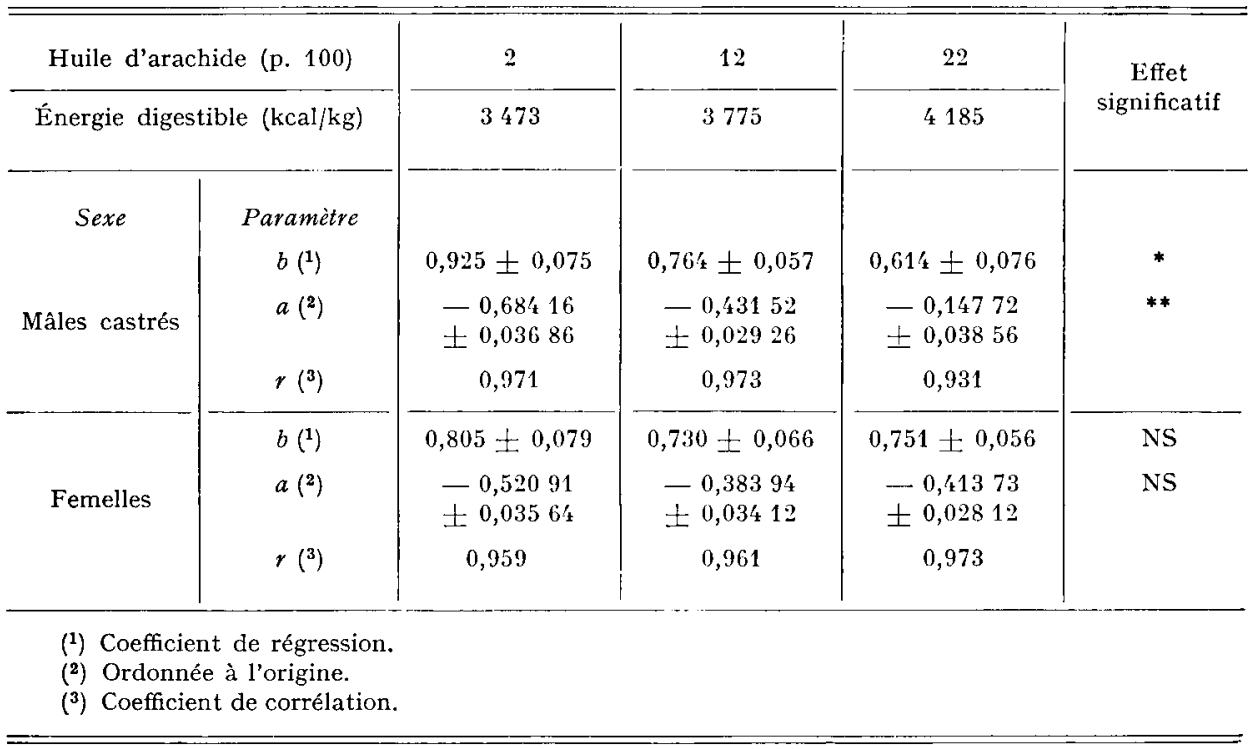


ressort que chez les mâles castrés le profil de la consommation d'énergie varie en fonction de sa concentration dans le régime : la consommation d'énergie pendant la première phase de la croissance est d'autant plus forte que la valeur énergétique de la ration est elle-même plus élevée, alors que le phénomène inverse est observé au cours de la période de finition. Contrairement aux mâles castrés, les femelles ajustent, à un poids donné, leur consommation d'énergie en fonction du taux d'incorporation d'huile d'arachide.

Le calcul de la régression de la consommation d'énergie (ED) sur le poids vif moyen $(\mathrm{P})$, pour l'ensemble des animaux de chaque sexe, conduit aux équations suivantes :

mâles castrés :

$\log \mathrm{ED}, \mathrm{Mcal} / \mathrm{j}=(-0,4 \mathrm{I} 47 \pm 0,04 \mathrm{I} 47)+(0,764 \pm 0,048) \log \mathrm{P}, \mathrm{kg}$;

femelles :

$r=0,942$,

$\log \mathrm{FD}, \mathrm{Mcal} / \mathrm{j}=(-0,4379 \pm 0,03 \mathrm{IgI})+(0,76 \mathrm{I} \pm 0,038) \log \mathrm{P}, \mathrm{kg}$;

$$
r=0,963 \text {. }
$$

Les valeurs des coefficients de régression étant voisines de 0,75 , il a été procédé, dans un deuxième temps, à un calcul de la régression de la consommation journalière d'énergie sur le poids métabolique, $\mathrm{P}^{0,75}$, en $\mathrm{kg}$. Les ordonnées à l'origine étant elles-mêmes non significativement différentes de zéro, nous avons imposé aux courbes, la contrainte de passer par le point origine (TOMASSONE, I967), ce qui aboutit aux relations ci-après :

$$
\begin{aligned}
& \text { mâles castrés : } \mathrm{ED}, \mathrm{Mcal} / \mathrm{j}=\left(0,407 \pm 0,0 \text { 057) } \mathrm{P}^{0,75} ; \quad r=0,997\right. \\
& \text { femelles : } \mathrm{ED}, \mathrm{Mcal} / \mathrm{j}=(0,38 \mathrm{I} \pm 0,0045) \mathrm{P}^{0,75} ; \quad r=0,998
\end{aligned}
$$

La consommation d'énergie à un poids donné est ainsi de 6 p. Ioo plus faible chez les femelles que chez les mâles castrés. Comme le montre le tableau 5, les valeurs trouvées diffèrent assez peu des normes énergétiques fixées par le NRC (1968) pour des porcs des deux sexes, nourris à volonté d'un régime à base de maîs et de tourteau de soja; remarquons cependant que dans le cas des données du NRC, le coefficient de proportionnalité entre la consommation d'énergie et le poids métabolique n'est pas constant et subit une légère diminution à mesure que le poids vif augmente.

TABLEAU 5

\begin{tabular}{|c|c|c|c|c|}
\hline Poids vif, (P), $\mathrm{kg}$ & 20 & 40 & 60 & 80 \\
\hline $\mathrm{P}^{0,75}, \mathrm{~kg}$ & 9,5 & 15,9 & 21,6 & 26,7 \\
\hline Mâles castrés. & 3,9 & 6,5 & 8,8 & 10,9 \\
\hline Femelles ........ & 3,6 & 6,1 & 8,2 & 10,2 \\
\hline N. R. C. $(1968)\left({ }^{1}\right)$ & 4,3 & 6,6 & 8,5 & 10,2 \\
\hline
\end{tabular}

Évolution de la consommation d'énergie, en Mcal d'énergie digestible par jour, en fonction du poids vif, par référence aux normes du N.R.C. (1968)

(1) Compte non tenu du sexe. 


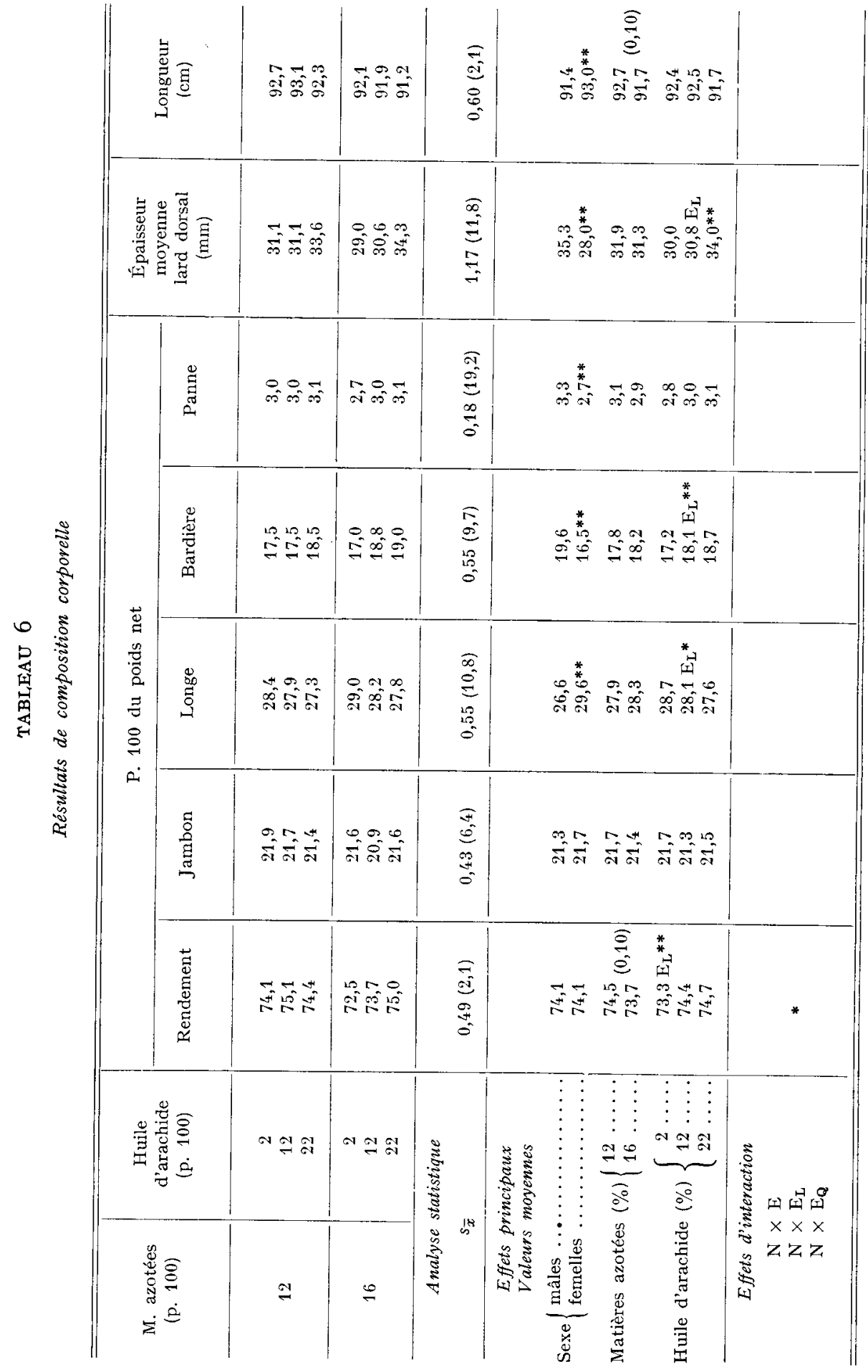




\section{B. - Composition corporelle}

Les résultats de composition corporelle sont indiqués dans le tableau 6. Les variations du rendement des carcasses font apparaître une interaction entre le taux de matières grasses et celui des matières azotées : l'élévation du pourcentage d'huile d'arachide est accompagnée d'une augmentation du rendement, mais seulement dans le cas des régimes à $\mathbf{I} 6 \mathrm{p}$. Ioo de matières azotées.

L'examen des données de la découpe des carcasses permet de retrouver l'effet habituel du sexe, qui se traduit par un état d'engraissement plus faible chez les femelles que chez les mâles castrés. L'élévation du taux d'incorporation d'huile d'arachide entraîne d'une façon systématique une diminution du pourcentage de longe dans la carcasse, en même temps qu'une augmentation du pourcentage de bardière et de l'épaisseur moyenne du lard dorsal.

En ce qui concerne le poids du contenu digestif, prélevé I6 heures environ après le dernier repas, et celui des viscères vides (tabl. 7), certains effets significatifs sont à noter. C'est ainsi que les femelles présentent un poids de contenu digestif plus

\section{TABLEAU 7}

Poids du contenu digestif et des viscèves

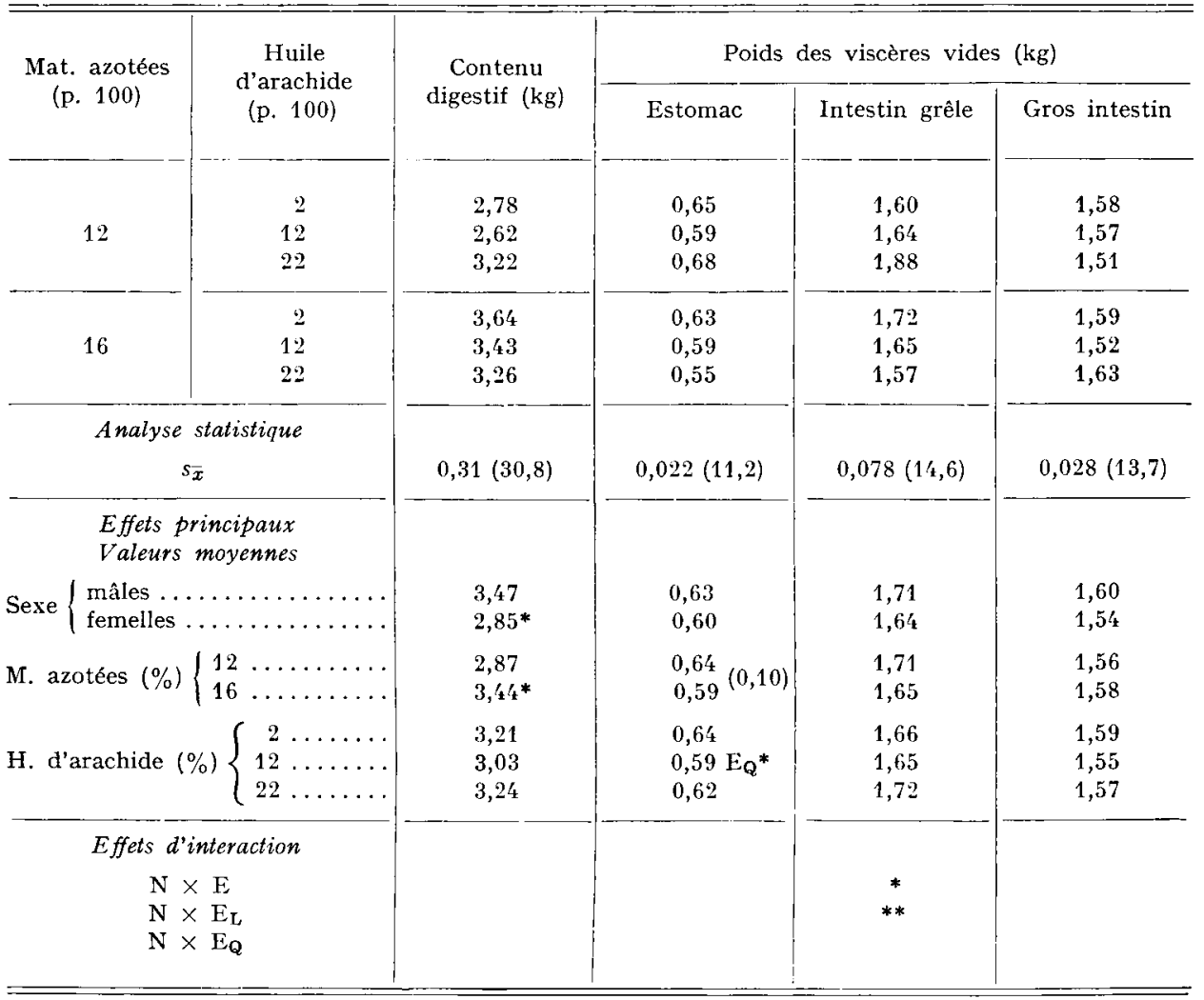


faible que les mâles castrés. Da la même façon, ce dernier est accru à la suite d'une élévation du taux de matières azotées de I 2 à I 6 p. Ioo, tandis que l'estomac vide aurait tendance à être plus lourd. L'évolution du poids frais de l'intestin grêle se traduit par une interaction entre le taux d'huile d'arachide (terme linéaire) et celui de matières azotées : pour une teneur de $\mathrm{I} 2 \mathrm{p}$. Ioo de ces dernières, le poids del'intestin grêle augmente avec la proportion d'huile d'arachide, alors que l'inverse a lieu au taux de $16 \mathrm{p}$. Ioo de matières azotées.

\section{DISCUSSION}

Les résultats de la présente étude ont permis de confirmer, tout au moins partiellement, les observations désormais classiques concernant les effets de l'élévation de la concentration en énergie du régime, par l'addition de matières grasses, sur les performances de croissance du Porc (HENRY et RÉRAT, I964). D'une manière générale, les données bibliographiques montrent que la diminution du niveau d'ingestion d'aliment est largement compensée par l'augmentation de sa valeur énergétique, de sorte que 1'apport quotidien d'énergie digestible est accru; il en résulte une amélioration de la vitesse de croissance, accompagnée d'ailleurs d'une augmentation de l'état d'engraissement des carcasses, lorsque le taux azoté du régime est maintenu constant.

Dans notre essai, un point mérite cependant d'être noté ; il s'agit de l'interaction entre le taux d'incorporation d'huile d'arachide et le niveau azoté de la ration. S'il apparaît que l'application d'un faible taux azoté, lorsqu'augmente le pourcentage de matières grasses, peut retentir défavorablement sur les performances, en raison précisément d'une ingestion azotée insuffisante pour une croissance optimum (CLAwsos, 1957), dans le cas présent, au contraire, c'est en élevant le taux de protéines de poisson de I2 à I $6 \mathrm{p}$. Ioo qu'on fait ressortir un effet dépressif du pourcentage d'huile d'arachide sur la croissance. La chute particulièrement importante du niveau d'ingestion de matières azotées ( 77 p. Ioo contre 7 au taux de I2 p. IOO) doit alors être rendue responsable de la diminution de la vitesse de croissance. Précisons toutefois qu'aucune explication satisfaisante ne peut être proposée pour justifier ce type inhabituel d'interaction entre les taux d'introduction d'huile d'arachide et de farine de poisson, mais déjà observé précédemment dans des conditions expérimentales analogues (HENRY et DE WILDE, I973).

L'évolution de la consommation en fonction du poids vif a fait apparaitre un comportement différent des animaux selon le sexe, en réponse à une modification de la concentration en énergie du régime. Le meilleur ajustement du niveau d'ingestion d'énergie observé chez les femelles, par rapport aux mâles castrés, s'explique par une aptitude plus grande à déposer du maigre au détriment du gras. On aboutit à la même constatation lorsque l'on examine les variations de la consommation spontanée d'énergie en fonction de l'apport azoté, dans le cas d'une distribution séparée d'un régime protidique, en quantité limitée, et d'un régime protéiprive à volonté (HENRY et RÉRAT, I968). Il est vraisemblable que cette capacité à limiter la consommation d'énergie varie également selon le type génétique, en relation avec l'intensité du développement musculaire. En tout état de cause, les résultats de notre étude mon- 
trent à l'évidence, tout au moins chez la femelle, que le niveau d'ingestion d'énergie, pour un poids donné, n'est pratiquement pas modifié par une élévation de la concentration en énergie de la ration de l'ordre de 20 p. Ioo au-delà de 3400 Kcalories d'énergie digestible $/ \mathrm{kg}$. Il est d'ailleurs sensiblement le même que celui défini par les normes du NRC, qui ont été établies pour un régime maïs-soja renfermant environ 3300 Kcalories d'énergie digestible $/ \mathrm{kg}$.

En ce qui concerne l'augmentation de l'état d'engraissement des animaux à l'abattage, consécutive à l'introduction d'huile d'arachide dans les régimes renfermant un taux constant de matières azotées, deux explications peuvent être données. L'accumulation des dépôts gras a d'abord été la conséquence d'une consommation d'énergie en excès par rapport à l'ingestion azotée, ce qui justifie le maintien d'un rapport azote-énergie constant dans le cas d'une variation de la concentration en énergie de la ration (CLAwson et al., I962). Sur ce point, nous confirmons 1'influence favorable d'un taux accru de protéines sur les performances pendant la phase initiale de croissance, déjà observée antérieurement (RÉRAT et HENRY, I964). Par ailleurs, les modifications quantitatives de la concentration en énergie des régimes n'ont pas été sans entraîner des changements dans la nature de l'énergie ingérée, qui intervient d'une manière spécifique au niveau du métabolisme lipidique. C'est ainsi que le remplacement de l'amidon de maïs par des proportions croissantes d'huile d'arachide, pour atteindre près de $40 \mathrm{p}$. Ioo de l'énergie digestible au taux le plus élevé ( $22 \mathrm{p}$. IOO), favorise l'état d'adiposité des carcasses en raison d'un dépôt préférentiel des acides gras longs de la source de matières grasses utilisée (FI,ANZY, FRANçoIs et RÉRAT, I970; NoRDSTROM et al., I972).

En définitive, l'augmentation de la concentration en énergie du régime au-delà de $345^{\circ}$ Kcalories d'énergie digestible/ $\mathrm{kg}$ et jusqu'à 4200 , par l'incorporation de taux croissants d'huile d'arachide, chez le porc en croissance nourri à volonté, provoque une diminution systématique du niveau d'ingestion d'aliment, tandis que la consommation d'énergie, à un poids donné, est sensiblement la même, quel que soit le pourcentage de matières grasses. Ces résultats indiquent qu'en dépit d'une forte concentration en énergie de la ration, le porc - la femelle plus encore que le mâle castré - est en mesure de maintenir sa capacité de régulation de son niveau d'ingestion énergétique.

Rę̧u pour publication en janvier 1974.

SUMMARY

INCORPORATION OF VARIABLE PROPORTIONS OF FAT (PEANUT OIL) INTO GROWING-FINISHING PIG DIETS, AS RELATED TO THE CRUDE PROTEIN CONTENT.

II. - EFFECT ON GROWTH PERFORMANCES AND BODY COMPOSITION

An experiment was carried out on 60 growing-finishing pigs of the Large White breed, between 25 and $90 \mathrm{~kg}$ live weight, in order to study the effect of variable proportions of dietary fat and protein on growth performances, feed intake, feed efficiency and body composition characteristics. The 6 treatments, each involving io animals (5 castrated males and 5 females), were applied according to a factorial scheme, $3 \times 2$, comprising 3 levels of peanut oil (2, I 2 and 22 p. Ioo and 2 levels of fish protein ( 12 and $16 \mathrm{p}$. IOO, in the form of Norvegian herring meal), in semipurified diets based on maize starch. The animals were fed to appetite and the diets were given wet. 
The findings showed that increase in the level of peanut oil from 2 to $22 \mathrm{p}$. Ioo, corresponding to a rise of the energy value of the diet from about $3400 \mathrm{kcal}$ of digestible energy/ $\mathrm{kg}$ to about $4200 \mathrm{kcal}$, led to a systematic lowering of the daily feed intake, more marked at a crude protein level of 16 p. Ioo than at 12 p. Ioo ( 19 p. Ioo versus 12 p. Ioo). On account of the increase in the energy value of the diets, the daily consumption of energy expressed as digestible energy, was slightly higher at a crude protein level of 12 p. Ioo ( + I 2 p. Ioo) and almost the same at I6 p. Ioo. This interaction between the level of peanut oil and that of fish protein was also found as regards the daily mean gain. The latter was not changed by the proportion of fat in the case of diets with i 2 p. Ioo crude protein, whereas at the level of $16 \mathrm{p}$. Ioo crude protein, it slightly decreased when the proportion of peanut oil increased from 2 to $22 \mathrm{p}$. Ioo. As regards the body composition, increasing incorporation of fat in the form of peanut oil, rich in long chain fatty acids, favoured the deposition of fatty tissues in the carcasses leading to increased feed efficiency (kcal digestible energy per $\mathrm{kg}$ weight gain), $i$. e. I I.o and $\mathrm{I} 2.0$ for 2 and $22 \mathrm{p}$. Ioo of peanut oil, respectively. From the variation in the energy intake according to live weight it appeared that, as compared to the males, the females showed a better adjustment of their ingestion level according to the energy content of the diet. The mean consumption of digestible energy, in mcal per $\mathrm{kg}$ metabolic weight $\left(P^{\mathbf{0 . 7 5}}\right)$, was higher in castrated males than in females $\left(0.4 \mathrm{I}\right.$ versus $\left.0.3^{8}\right)$.

\section{RÉFÉRENCES BIBLIOGRAPHIQUES}

Clawson A. J., Blumer T. N., Smart W. W., Jr, Barrick E. R., 1962. Influence of energy-protein ratio on performance and carcass characteristics of swine. J. Anim. Sci., 21, 62-68.

Clawson A. J., 1967. Influence of protein level, amino acid ratio and caloric density of the diet on feed intake and performance of pig. J. Anim. Sci., 26, 328-334.

Cochran W. G., Cox G. M., I962. Experimental designs. 6i I p., John Wiley and sons, New York. FISHER R. A., I958. Statistical methods for research workers, 356 p., Oliver and Boyd, London.

Flanzy J., François A. C., RÉrat A., r97o. Utilisation métabolique des acides gras chez le Porc. Ann. Biol. anim. Bioch. Biophys., 10, 603-620.

HenRy Y., Ig66. Variations des taux énergétique et azoté du régime chez le porc en croissance. $I X^{\mathrm{e}}$ Congrès Internat. de Zootechnie, Edinburg.

HENRY Y., DE WILDE R., I973. Incorporation de proportions variables de matieres grasses (huile d'arachide) dans le régime du Porc en croissance-finition, en relation avec le taux de matières azotées. I. Influence sur l'utilisation digestive des constituants énergétiques et azotés, et la rétention azotée. $A n n$. Zootech., 22, 167-184.

Henry Y., Rérat A., I964. Variation des taux énergétique et azoté dans l'alimentation du porc en croissance. Observations préliminaires. Ann. Biol. anim. Bioch. Biophys., 4, 263-27r.

Henry Y., Rérat A., I 968 . Libre ingestion de principes énergétiques chez le porc soumis à l'alimentation séparée. II Congrès mondial de Zootechnie, College Park, Maryland.

NRC (National Research Council), 1968. Nutrient requirements of swine. Pub. I599, National Academy of Sciences, Washington, DC.

Nordstrom J. W., Behrends B. R., Meade R. J., Thompson E. H., I972. Effects of feeding high oil corns to growing-finishing swine. J. Anim. Sci, 35, 357-36r.

Rérat A., Henry Y., 1964. Étude du besoin azoté chez le porc en croissance. I. Utilisation de la farine de poisson à trois taux différents. Ann. Zootech. 13, 5-34.

Tomassone R., 1967. Aperçu général sur les couches de croissance, i 8 p., Séminaire de Biométrie, Laboratoire de Biométrie, Nancy. 